Proceedings

\title{
The Vaginal and Fecal Microbiota associated to cervical cancer development in a mice model $^{\dagger}$
}

\author{
Fernando Hernández-Quiroz ${ }^{1}$, Selvasankar Murugesan ${ }^{1}$, Cristina Velazquez-Martínez ${ }^{1}$, Loan \\ Edel Villalobos-Flores 1, Otoniel Maya-Lucas ${ }^{1}$, Alberto Piña-Escobedo 1, Igrid García-González ${ }^{\text {, }}$ \\ Rodolfo Ocadiz-Delgado ${ }^{1}$, Paul F Lambert ${ }^{2}$, Patricio Gariglio ${ }^{1}$ and Jaime García-Mena ${ }^{1, *}$ \\ 1 Affiliation 1; Departamento de Genética y Biología Molecular, Centro de Investigación y de Estudios \\ Avanzados del Instituto Politécnico Nacional. Av IPN 2508 Col Zacatenco, Ciudad de México, 07360. \\ jgmena@cinvestav.mx \\ 2 Affiliation 2; McArdle Laboratory for Cancer Research, University of Wisconsin School of Medicine and \\ Public Health, Madison, WI 53706, USA. \\ * Correspondence: jgmena@cinvestav.mx. \\ + Presented at the $1^{\text {st }}$ International Electronic Conference on Microbiology, 2-30 November 2020; Available \\ online: https://ecm2020.sciforum.net/
}

Published: 02 November 2020

\begin{abstract}
Cervical cancer is a health issue worldwide. Many factors are related to this condition as persistent human papillomavirus infection, use of hormonal contraceptives, $\mathrm{pH}$ changes and bacterial vaginosis. The association between the microbiota and cervical cancer is an interesting issue; given that environmental and hormonal factors changing the vaginal microbiota may contribute to cancer. Using High-throughput DNA sequencing of V3-16S rDNA libraries, we determined the bacterial diversity in cervicovaginal lavages and fecal samples at different stages of cervical cancer development in the K14HPV16E7 mice model under $+/-17 \beta$-estradiol (E2) stimulus and compared it with an isogenic control (FVB). We found that continuous E2 administration during 6 months in the model with type 16 E7 expression causing development of cancer, is associated with significant changes in the microbiota diversity in the cervicovaginal lavages. Similar results were not observed in the model when no E2 was administered, neither in the FVB mice. Comparable changes in the microbiota diversity of fecal samples were not observed. The expression of E7 oncogene or the $17 \beta$-estradiol for longer periods of time, cause changes in the vaginal microbiota and the cervical epithelium not necessarily leading to cervical cancer; however, the action of both, contributes to cervical carcinoma.
\end{abstract}

Keywords: vaginal lavage; vaginal microbiota; fecal microbiota; cervical cancer; massive DNA sequencing

\section{Introduction}

Cervical cancer is the second most common cancer affecting woman worldwide [1], and several factors like the human papillomavirus (HPV) [2], the use of contraceptives [3], and the vaginal microbiota [4] contribute to this cancer. In humans, the vaginal microbiota protect against sexually transmitted infections, modulates immunity, and regulates $\mathrm{pH}$ [5]. Among the many commensal bacteria in the vagina, the lactobacillus are the more relevant species [6]. Persistent high-risk HPV type 16 and 18 infections are associated to $70 \%$ of cases [7]. HPV16 E6 and E7 oncogenes are factors for initiation and progression of cancer [8]. In our study we characterize the diversity of the vaginal and fecal bacterial microbiota in female mice after induction of cervical cancer, to understand the combined effect of HPV, E2 and the microbiota. We explored the existence of dysbiosis in the reproductive tract of the K14E7 female mice model during induction of cancer by $17 \beta$-estradiol. 


\section{Methodology}

\subsubsection{7 $\beta$-. estradiol treatment and induction of cancer}

The K14E7 [9] and the FVB [10] mice were housed and treated according to the AAALAC regulations. The Research Unit for Laboratory Animal Care Committee (NOM-062-ZOO-1999) approved all experimental procedure. For 17 $\beta$-estradiol treatment (E2) treatment, 1-month old virgin female transgenic and non-transgenic mice were implanted every month with continuous release pellets (Innovative Research of America, Cat. No. E-121) delivering $50.0 \mu \mathrm{g}$ of E2 for 60 days in the dorsal skin of 1-month old mice. Mice were sacrificed at month-6 of treatment (7-month-old) as previously described [11]. Subjects were classified in four groups: FvB (control), FvB+E2, K14E7, and K14E7+E2.

\subsection{Obtain of cervix tissue}

All the mice were sacrificed by cervical dislocation before dissection. All the samples were formalin-fixed, and paraffin embedded. Serial sections $5 \mu \mathrm{m}$ thick were processed for hematoxylin and eosin staining as previously reported [12].

\subsection{Detection the expression of transgene HPV16 E7}

For quality assurance of the appropriate expression of the E7 transgene in the K14E7 and FvB mice was confirmed the mRNA by qPCR as previously described [13].

\subsection{Sample collection}

Sample collection from the vaginal tract of female mice was made as previously described [14], with the following modifications: the cervicovaginal lavage was done by inserting a sterile pipette tip into the vaginal space flushing 3 times back and forth with $20 \mu \mathrm{L} 1 \mathrm{X}$ sterile phosphate-buffered saline (PBS). Fecal samples were collected directly from the mice in $1.5 \mathrm{~mL}$ sterile polypropylene tubes. All samples were frozen at $-80^{\circ} \mathrm{C}$ until further processing.

\subsection{DNA extraction from samples}

The genomic DNA from cervicovaginal was extracted from $200 \mu \mathrm{L}$ using QIAamp DNA Stool Mini Kit (QIAGEN) following the instructions. The DNA was eluted in $50 \mu \mathrm{L}$. The genomic DNA from fecal sample was extracted from $200 \mathrm{mg}$ of sample, DNA was extracted and eluted in 100. The quantity of purified DNA was measured at A260/280 ratio using a NanoDrop Spectrophotometer (Thermo Fisher Scientific, California USA), and the quality was evaluated by electrophoretic fractionation in $0.5 \%$ agarose gels. Average DNA concentration was $452.7 \mathrm{ng} / \mu \mathrm{L}( \pm 64.7 \mathrm{SD})$ for cervicovaginal lavage pools and $445.0( \pm 71.0 \mathrm{SD})$ for each fecal sample.

\subsection{Preparation of V3 $16 S$ rDNA libraries}

The libraries for sequencing were prepared for each cervicovaginal pool and fecal samples. Using an amplicon of $\sim 281 \mathrm{bp}$ containing the V3 variable region of the 16S RNA gene was amplified using V3-341F as previously described [15, 16]. PCR reactions were made using $50 \mathrm{ng}$ of template DNA in a final volume of $50 \mu \mathrm{L}$ as previously reported [16]. The libraries size and concentration were confirmed using the Agilent 2100 Bioanalyzer system.

\subsection{High-throughput DNA sequencing}

Sequencing was made using Ion OneTouch 2, Ion PGM Template OT2 200 Kit v2 DL (Life Technologies, California, USA), Ion 318 Chip Kit v2 and Ion Torrent PGM System as previously described [17]. After sequencing, all reads were filtered by the PGM software to exclude low quality and polyclonal sequences and were analyzed using FastQC software v0.11.9 [18] and trimmed to 200 nt using Trimmomatic v0.38. Demultiplexed FASTQ files were converted into FASTA files, 
concatenated into a single file, and then processed with multiple QIIME (Quantitative Insights into Microbial Ecology) v1.9.0 scripts [19]. DNA sequences were classified into Operational Taxonomic Units (OTUs) using closed based picking parameters with a $97 \%$ similarity level against Greengenes database v13.8. The sequence FASTQ files, and the corresponding mapping file for all samples used in this study, were deposited in the NCBI BioSample repository (Accession Number: PRJNA588243) link: https://www.ncbi.nlm.nih.gov/sra/PRINA588243.

\subsection{Determination of the abundance of the microbiota relative abundance}

Data were analyzed with QIIME pipeline to determine relative abundance of bacterial taxa. summarize_taxa_through_plots.py showed the relative abundance by plots.

\subsection{Determination of shared OTU's from microbiota}

We used the otu_table.biom files and the shared_phylotypes.py program from QIIME (v1.9.0) pipeline to determine the number of shared phylotypes between all the samples in cervicovaginal lavage and fecal samples.

\subsection{Statistical analyses}

All statistical analyses were done using SPSS (v24.0). Relative abundance values were analyzed using Mann-Whitney U tests.

\section{Results}

\subsection{Diferent profile of microbiota on cervicovaginal is associated to expression of the Type 16 E7 oncogene and/or E2 treatment}

The analyses of the relative abundance only show a tendency but not significant changes in the phyla abundances of the cervicovaginal lavages, comparing by the E2 treatment, there is an increase on the phylum of Firmicutes (p-value 0.26) and decrease of the phyla Bacteroidetes ( $p$-value 0.54) and Actinobacteria ( $p$-value 0.90) comparing K14E7 vs K14E7_E2; there is an increase of the phylum Proteobacteria ( $p$-value 0.46) and decrease of the phyla Firmicutes ( $p$-value 0.32) and Bacteroidetes ( $p$ value 0.71 ) when comparing FVB vs FVB_E2. In relation to E7 expression, K14E7 show more relative abundance of phyla Proteobacteria ( $p$-value 0.17), and Actinobacteria ( $p$-value 0.26), but lower relative abundance of the phyla Firmicutes ( $p$-value 0.38 ) and Bacteoridetes ( $p$-value 1.00) than the FVB mice; and K14E7_2 show more relative abundance of phylum Firmicutes ( $p$-value 0.21), but lower relative abundance of the phyla Proteobacteria ( $p$-value 0.90) and Actinobacteria ( $p$-value 1.00) than FVB_E2 (Fig. 1). There was not observed differences in the relative abundance for phyla in the fecal samples from same mice (Fig. 2).

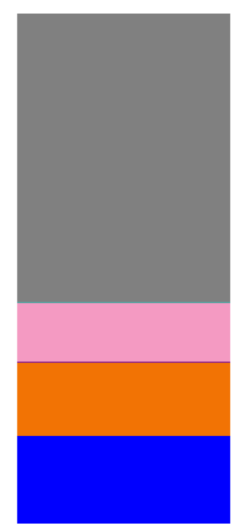

K14E7

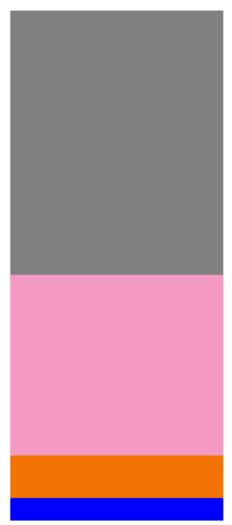

K14E7_E2

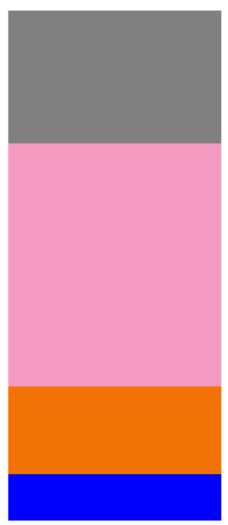

FVB

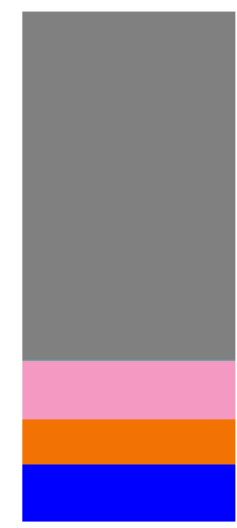

FVB E2
Proteobacteria Firmicutes

\section{Bacteroidetes}

- Actinobacteria

Figure 1. Relative abundance of bacterial phyla in cervicovaginal lavage of FVB and K14E7 mice. 


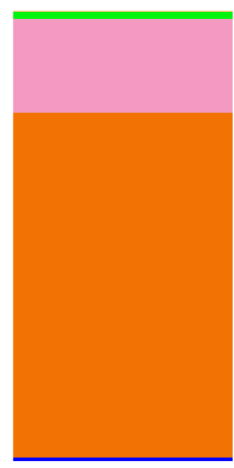

K14E7

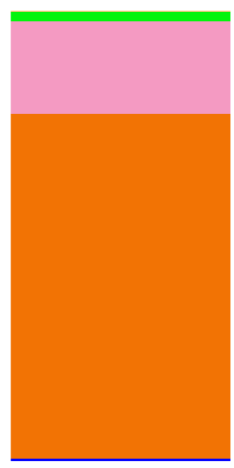

K14E7_E2

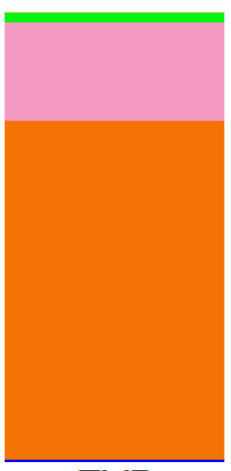

FVB

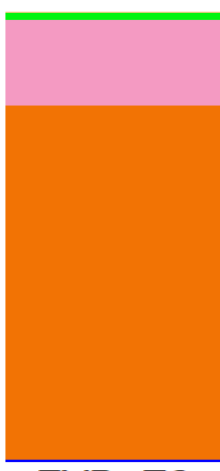

FVB_E2

Figure 2. Relative abundance of bacterial phyla in copro of FVB and K14E7 mice.

3.2. Samples from cervicovaginal have diferent number of OTU's shared due to the expression of the Type 16 E7 oncogene and/or E2 treatment

Using the shared phylotypes analyses e for the case cervicovaginal lavages, there are 7179 OTUs in common from K14E7 with or without E2, 3361 exclusive OTU's before the E2 treatment and 717 exclusive OTU's after E2 treatment on K14E7 mice; on other hand, there are 6091 OTUs in common from FVB with or without E2, 1149 exclusive OTUs before the E2 treatment and 2212 exclusive OTUs after E2 treatment on K14E7 mice (Fig. 3).

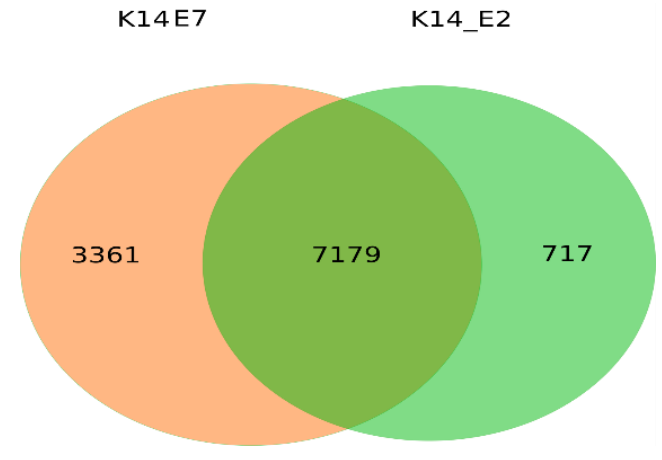

(a)

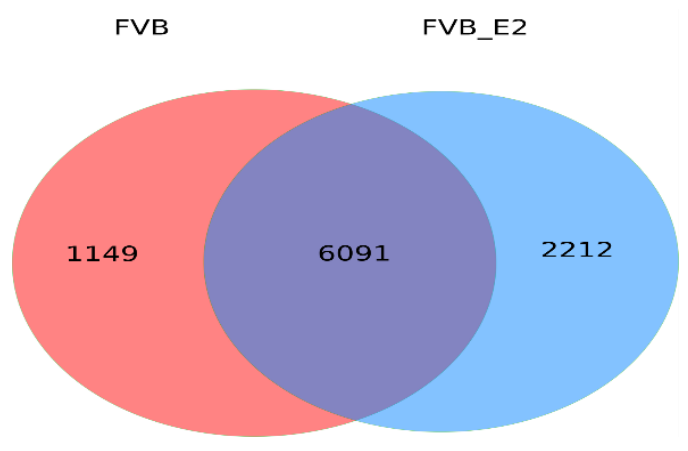

(b)

Figure 3. Shared OTU's from cervicovaginal lavage between K14E7 and FVB mice. (a) Comparing K14E7 with and without E2 treatment; (b) Comparing FVB with and without E2.

\section{Discussion}

In this study we characterized the abundance of phyla from the cervicovaginal and fecal microbiota in the K14E7 model for cervical cancer, with the objective to understand if there is synergia among Type 16 E7 oncogene expression, the use of $17 \beta$-estradiol, and changes in the composition of the vaginal microbiota. Our hypothesis was that changes in its diversity contribute to the development of cervical cancer. Although we not observed severe vaginal or fecal microbiota dysbiosis, we detected higher abundance of the phyla Proteobacteria on K14E7 with or without E2, and FVB with E2; this phylum is associated with cervical cancer [20].

\section{Conclusions}

We conclude that expression of the E7, or the use of hormonal contraceptives for instance 17 $\beta$ estradiol for longer periods of time, by their sides may cause changes on cervicovaginal microbiota but not necessarily this leads to cervical cancer; however, is necessary further studies for determine if the combined two factors can lead cervical carcinoma.

Author Contributions: Conceptualization, JG-M and P-G; methodology, CV-M, AP-E and S-M.; software, FHQ.; validation, JG-M, PF-L and P-G; formal analysis, FH-Q, IG-G, OM-L, LEV-F.; investigation, CV-M, S-M; resources, JG-M and P-G.; data curation, FH-Q, IG-G, OM-L, LEV-F; writing-original draft preparation, JG-M, 
IG-G, S-M and FH-Q; writing - review and editing, JG-M, IG-G, S-M and FH-Q; visualization, JG-M; supervision, JG-M and P-G; project administration, JG-M and P-G; funding acquisition, JG-M and P-G.

Funding: Work supported by Cinvestav, CONACyT 163235 INFR-2011-01, FONSEC SS/IMSS/ISSSTECONACYT-233361, and CONACyT 0201904 grants.

Acknowledgments: We thank Post-Doctoral Fellowship from FONSEC SS/IMSS/ISSSTE-CONACYT-233361 to SM; Post-Doctoral IG-G (398875), Doctoral OM-L (346907), LEV-F (336296), FH-Q (291236), and Master CV-M (553930) Fellowships from CONACyT. We are grateful to Alberto Piña-Escobedo, Rodrigo García-Gutiérrez, Lauro Macías, Ricardo Gaxiola-Centeno, Benjamin Chavez-Alvarez, and Rafael Leyva (UPEAL-Cinvestav, Mexico) for technical support, and Ms. Miriam Odet-Escobar Matamoros for administrative assistance.

Conflicts of Interest: The authors have no conflict of interest.

\section{References}

1. Salazar-Piña, D. A., Pedroza-Saavedra, A., Cruz-Valdez, A., Ortiz-Panozo, E., Maldonado-Gama, M., Chihu-Amparan, L., Rodriguez-Ocampo, A. N., Orozco-Fararoni, E., Esquivel-Guadarrama, F., \& Gutierrez-Xicotencatl, L. Validation of Serological Antibody Profiles Against Human Papillomavirus Type 16 Antigens as Markers for Early Detection of Cervical Cancer. Medicine, (2016) 95(6), e2769. https://doi.org/10.1097/MD.0000000000002769

2. Wardak S. Human Papillomavirus (HPV) and cervical cancer. Medycyna doswiadczalna $i$ mikrobiologia, (2016). 68(1), 73-84.

3. Asthana, S., Busa, V., \& Labani, S. Oral contraceptives use and risk of cervical cancer-A systematic review \& meta-analysis. European journal of obstetrics, gynecology, and reproductive biology, (2020). 247, 163-175. https://doi.org/10.1016/j.ejogrb.2020.02.014

4. Kovachev S. M. Cervical cancer and vaginal microbiota changes. Archives of microbiology, (2020). 202(2), 323327. https://doi.org/10.1007/s00203-019-01747-4

5. Kyrgiou, M., Mitra, A., \& Moscicki, A. B. Does the vaginal microbiota play a role in the development of cervical cancer?. Translational research : the journal of laboratory and clinical medicine, (2017). 179, 168-182. https://doi.org/10.1016/j.trsl.2016.07.004.

6. Martín, R., Soberón, N., Vaneechoutte, M., Flórez, A. B., Vázquez, F., \& Suárez, J. E. Characterization of indigenous vaginal lactobacilli from healthy women as probiotic candidates. International microbiology: the official journal of the Spanish Society for Microbiology, (2008). 11(4), 261-266. https://doi.org/10.2436/20.1501.01.70.

7. Ramakrishnan, S., Partricia, S., \& Mathan, G. Overview of high-risk HPV's 16 and 18 infected cervical cancer: pathogenesis to prevention. Biomedicine $\mathcal{E}$ pharmacotherapy = Biomedecine $\mathcal{E}$ pharmacotherapie, (2015). 70, 103-110. https://doi.org/10.1016/j.biopha.2014.12.041.

8. Popoca-Cuaya, M., Diaz-Chavez, J., Hernandez-Monge, J., Alvarez-Rios, E., Lambert, P. F., \& Gariglio, P. The HPV16 E6 oncoprotein and UVB irradiation inhibit the tumor suppressor TGF $\beta$ pathway in the epidermis of the K14E6 transgenic mouse. Experimental dermatology, (2015). 24(6), 430-435. https://doi.org/10.1111/exd.12689

9. Herber, R., Liem, A., Pitot, H., \& Lambert, P. F. Squamous epithelial hyperplasia and carcinoma in mice transgenic for the human papillomavirus type 16 E7 oncogene. Journal of virology, (1996). 70(3), 1873-1881. https://doi.org/10.1128/JVI.70.3.1873-1881.1996

10. Wong, P. K., Floyd, E., \& Szurek, P. F. High susceptibility of FVB/N mice to the paralytic disease induced by ts1, a mutant of Moloney murine leukemia virus TB. Virology, (1991). 180(1), 365-371. https://doi.org/10.1016/0042-6822(91)90041-9

11. Cortés-Malagón EM, Bonilla-Delgado J, Díaz-Chávez J, Hidalgo-Miranda A, Romero-Cordoba S, Uren A, Celik H, McCormick M, Munguía-Moreno JA, Ibarra-Sierra E, Escobar-Herrera J, Lambert PF, MendozaVillanueva D, Bermudez-Cruz RM, Gariglio P. Gene expression profile regulated by the HPV16 E7 oncoprotein and estradiol in cervical tissue. Virology, (2013). 447:155-65.

12. Ibarra Sierra, E., Díaz Chávez, J., Cortés-Malagón, E. M., Uribe-Figueroa, L., Hidalgo-Miranda, A., Lambert, P. F., \& Gariglio, P. Differential gene expression between skin and cervix induced by the E7 oncoprotein in a transgenic mouse model. Virology, (2012). 433(2), 337-345. https://doi.org/10.1016/j.virol.2012.08.024

13. Organista-Nava, J., Gómez-Gómez, Y., Ocadiz-Delgado, R., García-Villa, E., Bonilla-Delgado, J., LagunasMartínez, A., Tapia, J. S., Lambert, P. F., García-Carrancá, A., \& Gariglio, P. The HPV16 E7 oncoprotein 
increases the expression of Oct3/4 and stemness-related genes and augments cell self-renewal. Virology, (2016). 499, 230-242. https://doi.org/10.1016/j.virol.2016.09.020

14. Barfod, K. K., Vrankx, K., Mirsepasi-Lauridsen, H. C., Hansen, J. S., Hougaard, K. S., Larsen, S. T., Ouwenhand, A. C., \& Krogfelt, K. A. The Murine Lung Microbiome Changes During Lung Inflammation and Intranasal Vancomycin Treatment. The open microbiology journal, (2015). 9, 167-179. https://doi.org/10.2174/1874285801509010167

15. Fierer N, Hamady M, Lauber CL, Knight R. The influence of sex, handedness, and washing on the diversity of hand surface bacteria. Proc Natl Acad Sci, (2008). 105:17994-17999. https://doi.org/10.1073/pnas.0807920105

16. Murugesan, S., Ulloa-Martínez, M., Martínez-Rojano, H., Galván-Rodríguez, F. M., Miranda-Brito, C., Romano, M. C., Piña-Escobedo, A., Pizano-Zárate, M. L., Hoyo-Vadillo, C., \& García-Mena, J. Study of the diversity and short-chain fatty acids production by the bacterial community in overweight and obese Mexican children. European journal of clinical microbiology \& infectious diseases : official publication of the European Society of Clinical Microbiology, (2015). 34(7), 1337-1346. https://doi.org/10.1007/s10096-0152355-4

17. Hernández-Quiroz, F., Nirmalkar, K., Villalobos-Flores, L. E., Murugesan, S., Cruz-Narváez, Y., RicoArzate, E., Hoyo-Vadillo, C., Chavez-Carbajal, A., Pizano-Zárate, M. L., \& García-Mena, J. Influence of moderate beer consumption on human gut microbiota and its impact on fasting glucose and $\beta$-cell function. Alcohol (Fayetteville, N.Y.), (2020). 85, 77-94. https://doi.org/10.1016/j.alcohol.2019.05.006

18. Andrews S. (2010). FastQC: a quality control tool for high throughput sequence data. Available online at: http://www.bioinformatics.babraham.ac.uk/projects/fastqc).

19. Caporaso, J. G., Kuczynski, J., Stombaugh, J., Bittinger, K., Bushman, F. D., Costello, E. K., Fierer, N., Peña, A. G., Goodrich, J. K., Gordon, J. I., Huttley, G. A., Kelley, S. T., Knights, D., Koenig, J. E., Ley, R. E., Lozupone, C. A., McDonald, D., Muegge, B. D., Pirrung, M., Reeder, J., ... Knight, R. QIIME allows analysis of high-throughput community sequencing data. Nature methods, (2010). 7(5), 335-336. https://doi.org/10.1038/nmeth.f.303

20. Wang, Z., Wang, Q., Zhao, J., Gong, L., Zhang, Y., Wang, X., \& Yuan, Z. Altered diversity and composition of the gut microbiome in patients with cervical cancer. AMB Express, (2019). 9(1), 40. https://doi.org/10.1186/s13568-019-0763-z. 